\title{
Designing Visual Communication of Everyday IIIness Experiences in Pediatric Care
}

\author{
Matthew K. Hong \\ Georgia Institute of Technology \\ Atlanta, GA, USA \\ mhong@gatech.edu
}

\begin{abstract}
In complex chronic care, patients' ongoing awareness of their health status and ability to articulate health needs are vital to active participation in care, yet they face various challenges that could thwart their potential to engage in such participation. My research explores how design methods in $\mathrm{HCl}$ can evolve to meet these challenges by engaging both adolescents and family caregivers throughout the process of tracking the patients' illness experiences and co-designing rich representations that are expected to support adolescents' communication of these experiences in care. This thesis will contribute 1) a critical understanding of the ways in which human-centered design can address primary challenges that adolescents face when engaging in care, 2) a novel method for conducting codesign research with chronically ill patient families, and a 3) family-centered mobile health technology that demonstrates the feasibility of engaging pediatric patient families.
\end{abstract}

\section{CCS CONCEPTS}

- Human-centered computing $\rightarrow \mathrm{HCI}$ design and evaluation methods;

Permission to make digital or hard copies of part or all of this work for personal or classroom use is granted without fee provided that copies are not made or distributed for profit or commercial advantage and that copies bear this notice and the full citation on the first page. Copyrights for third-party components of this work must be honored. For all other uses, contact the owner/author(s)

CHI'19 Extended Abstracts, May 4-9, 2019, Glasgow, Scotland UK

(c) 2019 Copyright held by the owner/author(s).

ACM ISBN 978-1-4503-5971-9/19/05.

https://doi.org/10.1145/3290607.3299068 
${ }^{1}$ Complex Chronic Conditions: Complex chronic conditions (CCC), in its working definition in clinical studies, include a range of diseases that "can be reasonably expected to last at least 12 months and to involve either several different organ systems or one system severely enough to require specialty pediatric care and probably some period of hospitalization in a tertiary care center" [5]. By this definition, CCCs are different from more prevalent forms of pediatric chronic illnesses such as obesity and diabetes. Pediatric rheumatism, cancer, and blood disorders, which are the focus of my work, fall under this definition based on Feudtner et al.'s characterization.

${ }^{2}$ Patient-Generated Health Data: PatientGenerated Health Data (PGHD) are healthrelated data that are created or inferred by patients or care partners to help address a health concern. Two types of PGHD inform health care: clinician-, and patient-defined PGHD [2]. Clinician-defined PGHD are assessments that professionals find important, but that must be gathered by the person experiencing daily life to contribute to those assessments. On the other hand, patient-defined PGHD reflect concepts that are uniquely defined and seen as important by the patient.

${ }^{3}$ Observations of Daily Living: Observations of Daily Living (ODLs) is a conceptual framework and a specific type of patientdefined PGHD that describes PGHD reflecting the patients' perspective [2]. ODLs reflect feelings, thoughts, behaviors, exposures and actions that serve as cues to healthy action and indicators of personal health states.

Sidebar 1: Key Concepts and Background

\section{KEYWORDS}

mobile health; chronic illness; patient-generated health data; co-design; adolescent; family.

\section{CONTEXT AND MOTIVATION}

Design research in $\mathrm{HCl}$ has played a fundamental role in advancing the design of health technologies for chronically ill patients, yet research involving pediatric patients with special health care needs has been limited to date. In care of these patients, decisions about treatment and supportive care are made based on a comprehensive understanding of the patient's health data, including physiological data, physician's global assessment, and caregiver- and patient-reported observations of the patient's health status [3]. It is important that patients articulate their physical, psychological, social, and emotional well-being to the care team, but adolescent (ages 10-19) patients with CCC's ${ }^{1}$, in particular, face difficulty expressing these illness needs as their transition into adulthood marks an important yet challenging period of biopsychosocial development [12]. While they have the firsthand experience, there is little consideration for the patients' own assessments of their health in clinical decision making due to their dependence on family and clinical caregivers, conflicting interpretations about their health status, poor recall, and lack of means to understand and express their health needs.

To communicate these illness needs, patients will first need to build on meaningful representations of their everyday illness experience to support ongoing awareness of their health status [9]. When eliciting everyday illness experiences, digital tools that embrace visual storytelling can provide a powerful means to engage young people in their daily living $[4,11]$. It is now possible to tap into adolescents' latent potential to construct rich narratives of their illness experiences-powered by a suit of media technology that are readily accessible through personal mobile devices. Almost $95 \%$ of people in this age group today have access to a mobile device, and more than half who stay online have experience sharing their own digital creations, such as artwork, photos, stories, or videos through popular social media platforms [1]. Still, no tools exist to support their engagement and communication of illness needs that build upon these representations. My answer to this problem and broader research goal is to re-envision design methods to create collaborative health technologies that scaffold patients' gradual participation in care as designers-by means of engaging family members as co-design partners.

For my dissertation research, I am working with oncology, hematology, and rheumatology clinical collaborators at Children's Healthcare of Atlanta (CHOA) to understand how design methods can evolve to better engage adolescents and family caregivers throughout the process of tracking and co-designing rich representations of the patients' daily illness experiences that have personal relevance to their management of treatment effects. 
RQ 1: How do adolescents with CCC's and family caregivers participate in care? [7,8]

- Empirical analysis of patient-parent perspectives toward their participation in complex care prior to, and after being exposed to patient portal technology.

- Characterization of challenges and design space to support adolescents' participation in complex care.

RQ 2: How can we design tools to elicit in formation about adolescents' everyday illness experiences? [6, 10]

- Creation and expansion of co-design artifacts (Visual ODLs) and methodological innovation (scaffolding technique).

- Formative usability evaluation of UIs integrating Visual ODLs for clinical use.

RQ 3: How can we engage adolescent patients undergoing complex treatment in the design process, to create illness representations that are meaningful to these patients and their family members?

- Empirical analyses of interviews and diary entries, participant-generated artifacts from co-design, and quantitative EMA and survey measures.

- Characterization of technology affordances and family-tracking practices in capturing ODL data.

- Creation of novel research method: Episode-based Co-Design (ECD).

- Design and deployment of novel familyoriented mHealth application (CO-OP).

Sidebar 2: Research Questions and Summary of Contributions (bullets).

\section{COMPLETED AND ONGOING WORK}

For the past four years, I have been leading various research studies involving chronically ill adolescent patients in both clinical and non-clinical settings that span participant observations, interviews, portal usage log analysis, surveys, co-design, and a probe study. Thus far, I have completed two projects and started collecting data for the final project. Three research questions (Sidebar 2) guide my work.

\section{Understanding Patient Participation in Complex Care}

My early formative work provides an overview of the problem and design space for adolescents' participation in complex care, which address RQ 1. Through 38 individual interviews (in private) with patients, their parents, and the oncology team, I learned that adolescents' faced challenges participating in clinical conversations, communicating emotionally sensitive information, and managing physical and emotional responses. In particular, while patients downplayed their symptoms not to worry their parents, symptom reporting was often assumed by parental caregivers, even if the reported data may not adequately represent the patient's true felt experience [8].

In another study, I conducted log analysis, survey and interviews to understand adolescent and parent perspectives after they have navigated the patient's health information through a tethered personal health records system, yet found that current patient portal technologies had limited means to engage these patients in care [7]. These studies created contours of a design space for accommodating patient and parent perspectives in symptom reporting by supporting their gradually evolving partnerships through the design of a patient-friendly and engaging technology.

\section{Characterizing Patient-Defined Patient-Generated Data}

Informed by research on the positive impact of images on symptom communication [9] and the concept of Observations of Daily Living (ODLs ${ }^{3}$ ), my recent work, addressing $\mathbf{R Q} \mathbf{2}$, explored the use of co-design methods to characterize patient-defined PGHD $^{2}$ for communicating adolescents' lived experience [6]. In this work I adapted the ODL framework and solicited domain knowledge from 11 clinicians to generate design artifacts, or Visual ODLs (Fig. 1), to elicit everyday illness experiences through co-design sessions with 14 patient families. Through co-design, I found the need for technology to scaffold the process of encoding and articulating symptomatic experiences through representation of how symptoms affect patients' ability to engage in daily activities, to allow full expression of these experiences through the use of varied forms of media data representations, and to support distinct roles which family caregivers can serve in tracking the patient experience (e.g., timing and frequency). In another study, I interviewed 15 clinicians in an onco-hematology setting to examine clinicians' perspectives on potential use cases for incorporating Visual ODLs through tablet displays in their practice. In specific, they promoted patient-initiated first-person illness narratives 


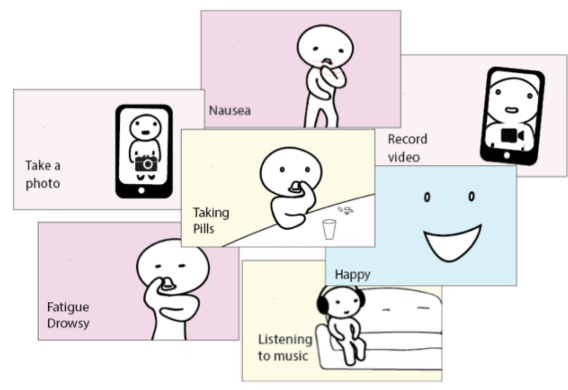

Figure 1: Visual Observations of Daily Living (ODLs). Visual ODLs are co-design artifacts that represent multiple aspects of the patients' daily illness experiences ranging from physical and emotional symptoms, daily activities, and social and environmental contexts.

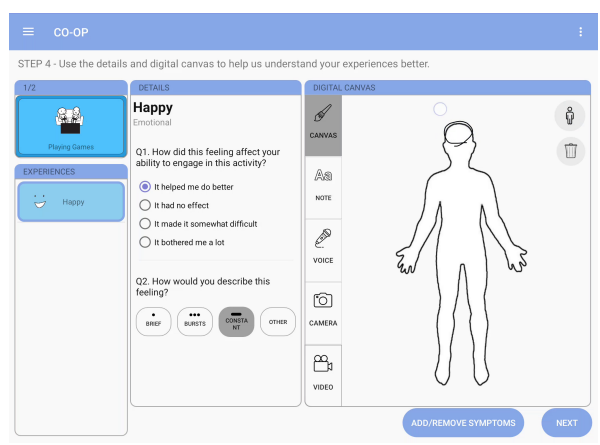

Figure 2: CO-OP mHealth system. Visual ODLs are being used to elicit daily illness experiences and design feedback from patients and family caregivers. during face-to-face encounters, and valued a summarized view of the patient's illness experience between visits that highlights concerning symptoms by presenting symptom attributes in the order of highest frequency, severity and interference with specific daily activities [10]. These insights informed the design of the current CO-OP system (Fig. 2) and research goal to understand how the family could work together as co-designers while tracking the patients' health.

\section{Supporting Situated Design Work in Everyday Life}

I am currently drawing on design methods to create an interactive mHealth system as an endpoint of the novel design process that investigates how technology can support patients and family caregivers' collaborative effort to track and co-create personally meaningful representations of everyday illnesss experiences. Over a 2-3 week period, the system will elicit and probe patients' and family caregivers' observations of symptomatic experiences in relation to their everyday activities, and their design input, through a suit of media technology readily available on their mobile device. One goal of this work is to create and evaluate a new method, 'Episode-Based Co-Design (ECD)', which is motivated by the lack of methods for engaging patients and family members in the co-design process when the design needs to be close in time to phenomena of interest (e.g., in this case, an episode of the illness experience).

To address RQ 3, I apply the ECD method to adolescent patients with CCC's, as a case study, to show that the method is useful, valid, and reliable for its intended purpose [13]. To do this, I employ ecological momentary assessment (EMA) methods that are geared towards achieving high ecological validity by placing the data collection activities in the hands of patients and their parental caregivers, in their natural setting. To better understand patient and parents' conceptions of the illness experience, I am conducting co-design activities that are informed by a rich set of data collaboratively collected on a daily basis by the patient family. To measure the relevance of illness representations, co-designed artifacts will be assessed by a subgroup of patient populations belonging to similar disease categories. Through this research, I aim to analyze many factors with respect to patients' engagement in care, including compliance, quantity and quality of design outcomes, distribution of tracking tasks among family members, and the unique privacy needs arising from family-based tracking.

My work is expected to inform the design of tools that support patient and family partners' engagement with their everyday lived experiences by scaffolding their co-creation of personally meaningful representations. These co-designed representations could support other vulnerable populations who rely on a network of caregivers to better articulate their experiences. 


\section{ACKNOWLEDGEMENTS}

I would like to thank GVU/IPaT and NSF for their funding and support. I would also like to thank my advisor, Professor Lauren Wilcox, and collaborators at Georgia Tech. Finally, I would like to thank all my research partners and study participants at the Children's Healthcare of Atlanta.

\section{REFERENCES}

[1] [n. d.]. Teen Content Creators and Consumers | Pew Research Center. http://www.pewinternet.org/2005/11/02/ teen-content-creators-and-consumers/

[2] Patricia Flatley Brennan and Gail Casper. 2015. Observing health in everyday living: ODLs and the care-between-the-care. Personal and Ubiquitous Computing 19, 1 (1 2015), 3-8. https://doi.org/10.1007/s00779-014-0805-0

[3] Prasanna R Deshpande, Surulivel Rajan, B Lakshmi Sudeepthi, and C P Abdul Nazir. 2011. Patient-reported outcomes: A new era in clinical research. Perspectives in clinical research 2, 4 (10 2011), 137-44. https://doi.org/10.4103/2229-3485.86879

[4] Sarah E. Drew, Rony E. Duncan, and Susan M. Sawyer. 2010. Visual Storytelling: A Beneficial But Challenging Method for Health Research With Young People. Qualitative Health Research 20, 12 (12 2010), 1677-1688. https://doi.org/10.1177/ 1049732310377455

[5] Chris Feudtner, Ross M Hays, Gerri Haynes, J Russell Geyer, John M Neff, and Thomas D Koepsell. 2001. Deaths attributed to pediatric complex chronic conditions: national trends and implications for supportive care services. Pediatrics 107, 6 (2001), e99-e99.

[6] Matthew K. Hong, Udaya Lakshmi, Thomas A. Olson, and Lauren Wilcox. 2018. Visual ODLs: Co-Designing PatientGenerated Observations of Daily Living to Support Data-Driven Conversations in Pediatric Care. In Proceedings of the 2018 CHI Conference on Human Factors in Computing Systems - CHI '18. ACM Press, New York, New York, USA, 1-13. https://doi.org/10.1145/3173574.3174050

[7] Matthew K Hong, Lauren Wilcox, Clayton Feustel, Karen Wasilewski-Masker, Thomas A Olson, and Stephen F Simoneaux 2016. Adolescent and Caregiver use of a Tethered Personal Health Record System. In AMIA Annual Symposium Proceedings. American Medical Informatics Association, Chicago.

[8] Matthew K. Hong, Lauren Wilcox, Daniel Machado, Thomas A. Olson, and Stephen F. Simoneaux. 2016. Care Partnerships. In Proceedings of the 2016 CHI Conference on Human Factors in Computing Systems - CHI '16. ACM Press, New York, 5337-5349. https://doi.org/10.1145/2858036.2858508

[9] Peter S. Houts, Cecilia C. Doak, Leonard G. Doak, and Matthew J. Loscalzo. 2006. The role of pictures in improving health communication: A review of research on attention, comprehension, recall, and adherence. Patient Education and Counseling 61, 2 (5 2006), 173-190. https://doi.org/10.1016/J.PEC.2005.05.004

[10] Udaya Lakshmi, Matthew Hong, and Lauren Wilcox. 2018. Integrating Patient-Generated Observations of Daily Living into Pediatric Cancer Care: A Formative User Interface Design Study. In 2018 IEEE International Conference on Healthcare Informatics (ICHI). IEEE, 265-275. https://doi.org/10.1109/ICHI.2018.00037

[11] Michael Rich, Steven Lamola, Jason Gordon, and Richard Chalfen. 2000. Video intervention/prevention assessment: a patient-centered methodology for understanding the adolescent illness experience. Journal of Adolescent Health 27, 3 (9 2000), 155-165. https://doi.org/10.1016/S1054-139X(00)00114-2

[12] Susan M Sawyer, Sarah Drew, Michele S Yeo, and Maria T Britto. 2007. Adolescents with a chronic condition: challenges living, challenges treating. The Lancet 369, 9571 (2007), 1481-1489.

[13] Jacob O. Wobbrock and Julie A. Kientz. 2016. Research Contributions in Human-Computer Interaction. interactions 23, 3 (4 2016), 38-44. https://doi.org/10.1145/2907069 